\title{
Small bowel obstruction due to internal herniation through a defect in the falciform ligament: a rare case report and review of the literature
}

\author{
Habib Ahmad Esmat ${ }^{1,2^{*}}$ and Mohammad Wali Naseri ${ }^{3}$
}

\begin{abstract}
Background: The falciform ligament hernia is a type of internal herniation, caused by a defect in the falciform ligament of the liver. The etiology for this defect may be congenital or iatrogenic, created after the laparoscopic intervention.

Case presentation: A 45-year-old male was presented to our hospital, complaining of abdominal pain for 2 days. The physical examination revealed diffuse abdominal tenderness and guarding. The abdominal X-ray was suggestive of intestinal obstruction. On abdominal CT images, dilated proximal ileal loops between the left hepatic lobe and anterior abdominal wall, forming a closed-loop with mesenteric edema and fat stranding, were observed. There were findings of ileus in the jejunal loops proximal to this segment, but the ileal loops and the colon were completely collapsed, consistent with small bowel obstruction due to internal herniation through a defect in the falciform ligament. The patient underwent surgical intervention, reduction of the herniated bowel loops, and repairing the hernia defect. He had an uneventful recovery with a favorable outcome.

Conclusion: Herniation through a defect in the falciform ligament is extremely rare but should be considered in the differential diagnosis of acute abdomen. The clinical manifestations of falciform ligament hernia are nonspecific and may underestimate the diagnosis, leading to a delayed treatment that affects the management outcome. Computed tomography plays an important role in the timely diagnosis and planning of surgical intervention, precluding intestinal strangulation.
\end{abstract}

Keywords: Small bowel obstruction, Internal hernia, Falciform ligament, Case report

\section{Background}

A falciform ligament hernia is a herniation through an abnormal opening in the falciform ligament of the liver that usually contains the small bowel, though the omentum and colon have also been reported as hernia contents [1].

\footnotetext{
* Correspondence: habib.smt@gmail.com

'Department of Radiology, Kabul University of Medical Sciences, Kabul, Afghanistan

${ }^{2}$ Ege Universitesi Tip Fakultesi, Izmir, Turkey

Full list of author information is available at the end of the article
}

Herniation through the falciform ligament is extremely rare and the majority of cases are related to congenital defects involving malformation, hypoplasia, or complete failure of the development of the falciform ligament [2].

The clinical presentation is often nonspecific that may mimic biliary pathologies, peptic ulcer disease, and abdominal pain [3].

Inability to consider the primary etiology may result in delayed diagnosis and treatment, which increases morbidity and mortality [4].

Although the diagnostic laparoscopic approach is safe and associated with rapid patient recovery, Multidetector 
computed tomography (MDCT) plays an important role in preoperative diagnosis of internal hernias [3].

The authors present here an unusual case of small bowel obstruction due to internal herniation through a defect in the falciform ligament in a 45-year-old male.

\section{Case presentation}

A 45-year-old male patient was presented to our hospital, complaining of abdominal pain for 2 days. He had a history of hypertension and sleep apnea, but he did not give any history of food or drug allergy and psychosocial problems. In physical examination, diffuse abdominal tenderness and guarding were noted. Other findings were unremarkable. The laboratory analysis yielded hematocrit (Hct) $42 \%$, white blood cells $7700 / \mathrm{mm}^{3}$, and CRP $40.6 \mathrm{mg} / \mathrm{L}$. Abdominal X-ray was suggestive of intestinal obstruction (Fig. 1). The abdominal ultrasound exam revealed minimal free fluid in the peritoneal cavity. However, no occlusion was detected in mesenteric vessels on the Doppler exam. On abdominal CT images, dilated proximal ileal loops between the left hepatic lobe and anterior abdominal wall, forming a closed-loop with mesenteric edema and fat stranding, were observed. There were findings of ileus in the jejunal loops proximal to this segment, but the ileal loops and the colon were completely collapsed, consistent with small bowel obstruction due to internal herniation probably through a defect in the falciform ligament (Figs. 2, 3, and 4).

The patient was transferred to the general surgery unit for exploratory laparotomy as a part of treatment. On exploration, an ileal loop about $30 \mathrm{~cm}$ in length was herniating through approximately $2 \mathrm{~cm}$ congenital defect in

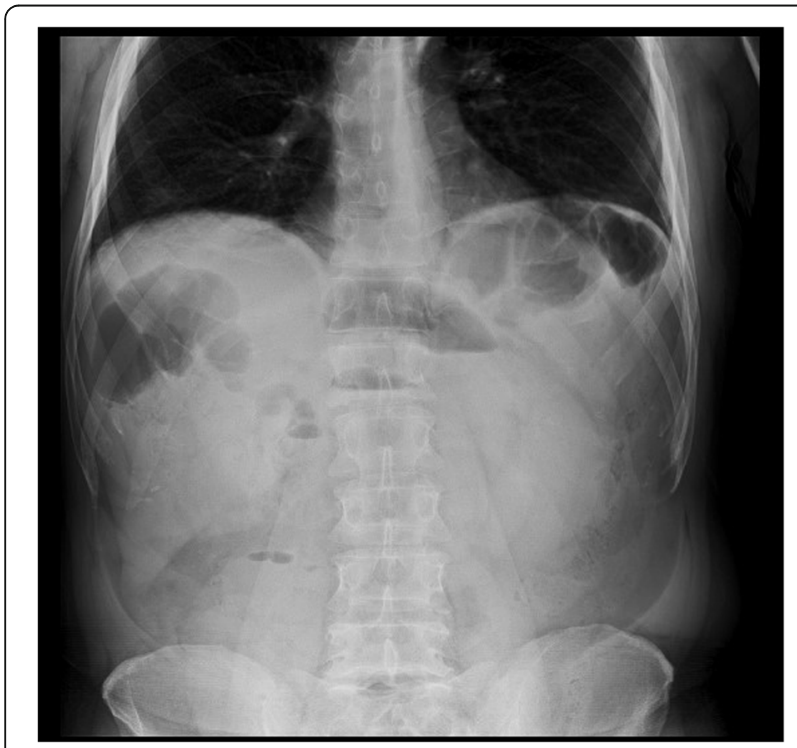

Fig. 1 Anteroposterior abdominal X-ray showing dilated bowl loop in the central region of the abdomen with an air-fluid level, consistent with small bowel obstruction the falciform ligament. The bowl loops seemed to be ischemic, but when the reduction was achieved, it was observed that its color improved and there was peristalsis. The falciform ligament defect was repaired, and the operation terminated without any complication. The patient had an uneventful recovery and discharged on postoperative day 4 , with a favorable outcome.

\section{Discussion}

Herniation through the falciform ligament is extremely rare, accounts for $0.2 \%$ of all internal hernias, and is often reported in early childhood or late pregnancy [2, 4]. Incomplete development and malformation of the falciform ligament may be the probable congenital etiology for the defects in this ligament [5]. However, most are iatrogenic, occurring after the laparoscopic intervention [4]. Excess visceral mobility and/or displacement into the upper abdomen are the important contributing risk factors [6].

The first case of falciform hernia was described in 1929 , and the increasing utility of laparoscopic exams increases each decade the number of new cases of this entity [3]. There are only individual case reports and case series in the literature search about the falciform ligament hernia through a congenital defect.

In a case series of 14 cases of internal hernias reported by Gullino et al., only two cases had hernias through an abnormal opening from the absence of the falciform ligament [5]. In our case, the defect in the falciform ligament seems to be congenital because the patient had never undergone abdominal surgical procedures.

The internal hernias are classified by Steinke into three groups: (I) intraperitoneal (transverse mesocolon, anomalous defect in the mesentery, omentum, and broad ligament), (II) retroperitoneal (paraduodenal, paracecal, intersigmoid, supravesical, and through the foramen of Winslow), and (III) postoperative. The falciform ligament hernia is included in the intraperitoneal type [7].

The clinical presentation is often nonspecific that may mimic biliary pathologies, peptic ulcer disease, and abdominal pain [3]. Inability to consider the primary etiology may result in delayed diagnosis and treatment, which increases morbidity and mortality [4].

Although the diagnostic laparoscopic approach is safe and associated with rapid patient recovery, multidetector computed tomography (MDCT) plays an important role in preoperative diagnosis of internal hernias, intestinal obstruction, and planning of surgical intervention $[4,8,9]$.

Repairing the hernia defect and division of the falciform ligaments and surgical management of bowel ischemia due to strangulation are the mainstay of treatment [6]. Though the internal hernia through a defect in the falciform ligament is very rare, it should always be considered 

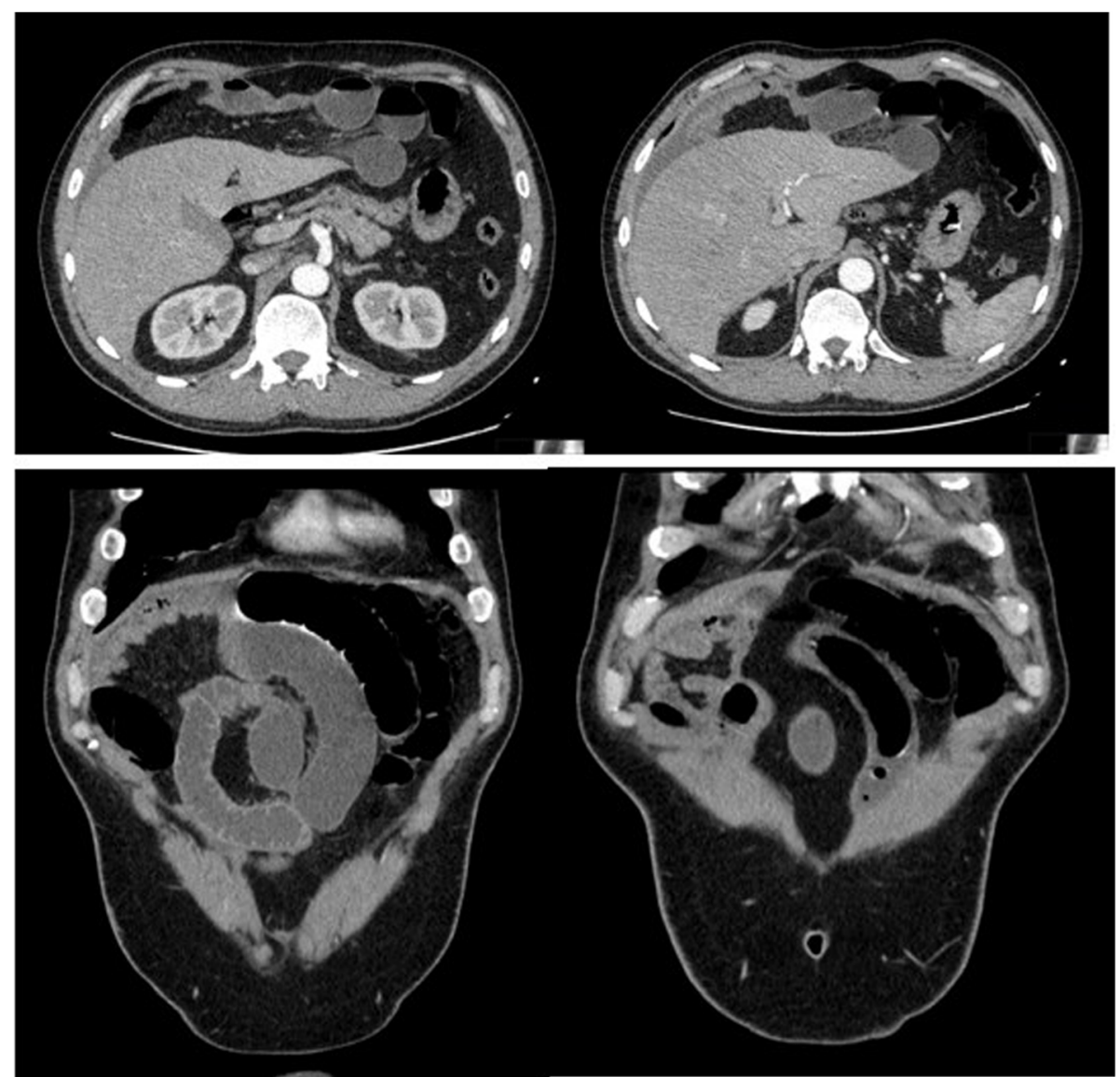

Fig. 2 Axial (a) and coronal (b) abdominal CT images showing dilated small bowel loops in two levels (arrows) between the anterior abdominal wall and left hepatic lobe, compatible with closed-loop obstruction through the falciform ligament

as a possibility because it has a very insidious onset and high mortality [10].

This is a rare and surgically proven case of internal herniation; however, the lack of long-time follow-up may be the only limitation for this case report.

\section{Conclusion}

Herniation through a defect in the falciform ligament is extremely rare but should be considered in the differential diagnosis of acute abdomen. The clinical manifestations of falciform ligament hernia are nonspecific and
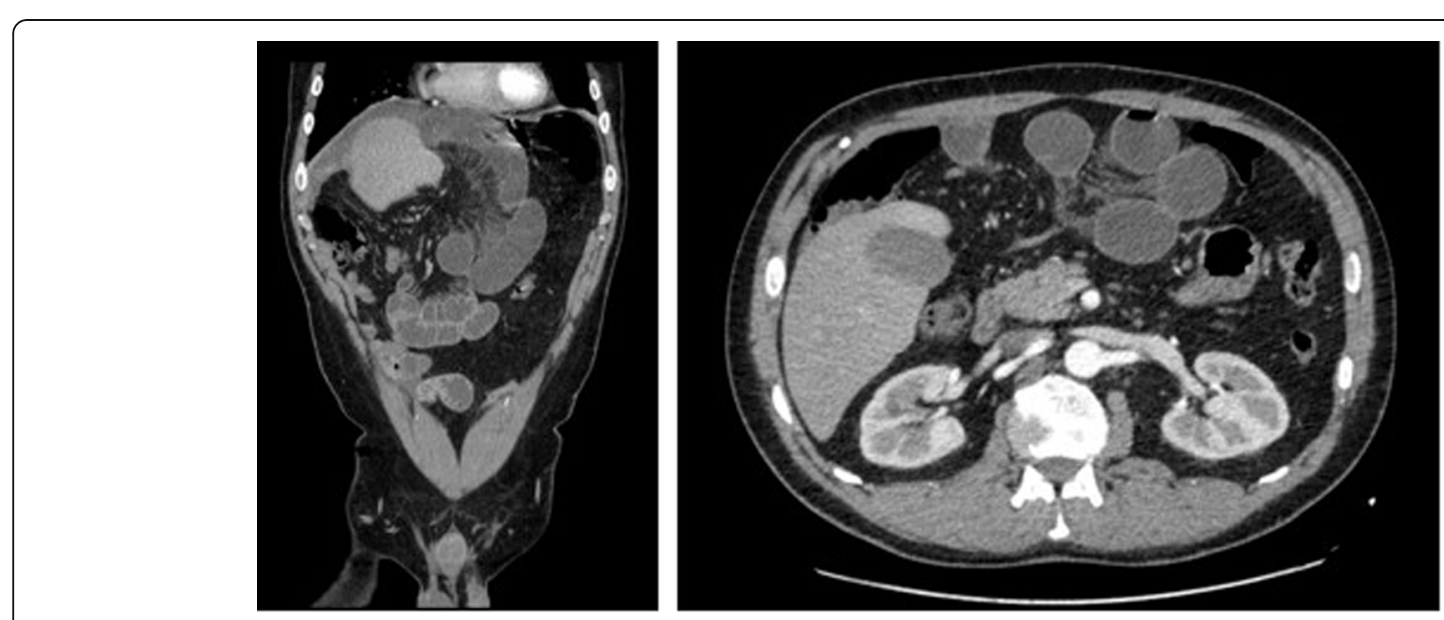

Fig. 3 Corona (a) and axial (b) abdominal CT images showing dilated small bowel loops with mesenteric congestion and fat stranding 


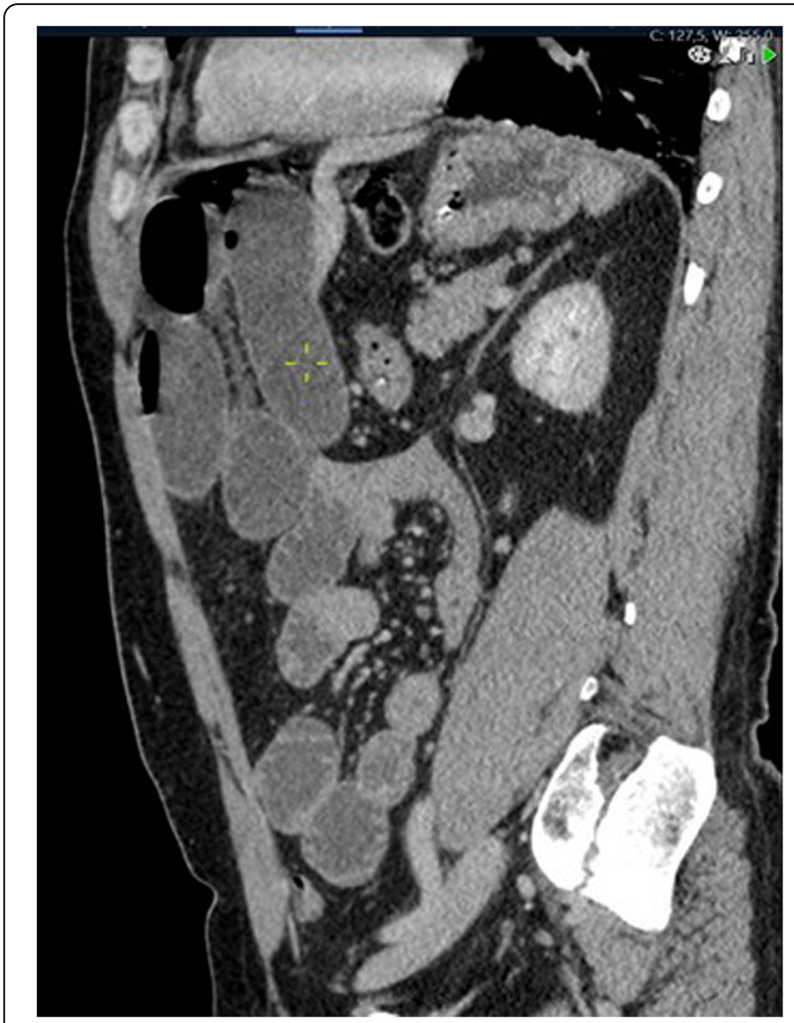

Fig. 4 Sagittal abdominal CT image showing dilated small bowel loops with mesenteric congestion and fat stranding

may underestimate the diagnosis, leading to a delayed treatment that affects the management outcome. Computed tomography plays an important role in the timely diagnosis and planning of surgical intervention, precluding intestinal strangulation.

\section{Abbreviations}

CT: Computed tomography; CRP: C-reactive protein; MDCT: Multidetector computed tomography

\section{Acknowledgements}

Not applicable.

\section{Authors' contributions}

Concept: HAE. Design: HAE. Supervision: MWN. Resources and data collection: HAE. Literature search: HAE. Writing of the manuscript: HAE. Critical review: MWN. All authors have read and approved the final manuscript.

\section{Funding}

We declare that our work is not funded by any institution, organization, or government, and we have no financial support.

\section{Availability of data and materials}

The data used during the current study are available from the corresponding author on reasonable request

\section{Declarations}

\section{Ethics approval and consent to participate}

The manuscript got an ethical review exemption from the Ethical Review Committee (ERC) of our hospital as case reports are exempted from review according to the institutional ethical review committee's policy.

\section{Consent for publication}

Written informed consent was obtained from the patient for publication of this case report and any accompanying images. A copy of the written consent is available for review by the editor of this journal.

\section{Competing interests}

The authors declare that they have no competing interests.

\section{Author details}

'Department of Radiology, Kabul University of Medical Sciences, Kabul, Afghanistan. ${ }^{2}$ Ege Universitesi Tip Fakultesi, Izmir, Turkey. ${ }^{3}$ Department of Internal Medicine, Kabul University of Medical Sciences, Kabul, Afghanistan.

Received: 5 February 2021 Accepted: 16 June 2021

Published online: 24 June 2021

\section{References}

1. Doishita S, Takeshita T, Uchima Y, Kawasaki M, Shimono T, Yamashita A, Sugimoto M, Ninoi T, Shima H, Miki Y (2016) Internal hernias in the era of multidetector $C$ : correlation of imaging and surgical findings. Radiographics. 36(1):88-106. https://doi.org/10.1148/rg.2016150113

2. Dusu K, Dindyal S, Gadhvi V (2015) Small bowel obstruction via herniation through an iatrogenic defect of the falciform ligament following laparoscopic cholecystectomy. Ann R Coll Surg Engl. 97(6):e93-e95. https:// doi.org/10.1308/rcsann.2015.0022

3. Macina S, Testa T, Losacco C (2016) Congenital internal hernia through defect in the falciform ligament in adult: a case report and review of the literature. Int J Surg Case Rep. 26:104-107. https://doi.org/10.1016/j.jscr.201 6.05.003

4. Ramakrishnaiah SB, Giridhar-Boggaram S, Paul-Satyaseela M (2015) Small bowel obstruction due to internal herniation of jejunum through a congenital defect in the falciform ligament in a primigravida. Arch Int Surg 5(1):46-48. https://doi.org/10.4103/2278-9596.153161

5. Lakdawala M, Chaube SR, Kazi Y, Bhasker A, Kanchwala A (2009) Internal hernia through an iatrogenic defect in the falciform ligament: a case report. Hernia. 13(2):217-219. https://doi.org/10.1007/s10029-008-0424-7

6. Wiseman S (2000) Internal herniation through a defect in the falciform ligament: a case report and review of the world literature. Hernia. 4(2):117$120 \mathrm{https}: / / \mathrm{doi} .0 \mathrm{rg} / 10.1007 / \mathrm{BF} 02353760$

7. Gaster J (1948) Internal hernia with strangulation of bowel due to a defect in the falciform ligament. Ann Surg. 128(2):248-252. https://doi.org/10.1097/ 00000658-194808000-00007

8. Parikh AP, Tyagi MP, Kapadia DA $(2019,2019)$ Intestinal obstruction in case of internal hernia through congenital defect in falciform ligament in adult. Int J Res Med Sci, DOI: https://doi.org/10.18203/2320-6012.jirms20195018 7(11):4368-4369

9. Monica ML, Antonella M, Gloria A, Diletta C, Nicola M, Ginevra D et al (2019) Internal hernias: a difficult diagnostic challenge. Review of $C T$ signs and clinical findings. Acta Biomed 90(5-S):20-37. https://doi.org/10.23750/abm. v90i5-S.8344

10. Özden Ö, Ekinci S (2014) Internal herniation through the falciform ligament: an unusual cause of intestinal obstruction. Turk J Pediatr. 56(5):551-552

\section{Publisher's Note}

Springer Nature remains neutral with regard to jurisdictional claims in published maps and institutional affiliations. 